\title{
INVARIANT METRICS IN GROUPS (SOLUTION OF A PROBLEM OF BANACH)
}

\author{
V. L. KLEE, JR. ${ }^{1}$
}

Introduction. If $G$ is a semi-group and $\rho$ a metric on $G, \rho$ will be called left invariant if $\rho(g x, g y)=\rho(x, y)$ whenever $\{g, x, y\} \subset G$, right invariant if always $\rho(x g, y g)=\rho(x, y)$, and invariant if it is both right and left invariant. If $T$ is a topological space and $\rho$ a metric on $T$, we shall say that $T$ admits $\rho$ if the $\rho$-topology of $T$ agrees with its original topology. G. Birkhoff [2] ${ }^{2}$ and Kakutani [5] proved that a Hausdorff group admits a left invariant metric if and only if it satisfies the first axiom of countability. $\$ 1$ below contains some remarks on invariant metrics, ${ }^{3}$ including a slight sharpening of the theorem just mentioned.

A topological space will be called topologically complete if it admits a metric under which it is complete. The principal result of this note (2.4) is that if $G$ is a Hausdorff group which is abelian, metrizable, and topologically complete, then $G$ admits an invariant metric under which it is complete. As applied to linear metric spaces, this answers affirmatively a question of Banach [1, p. 232].

I am indebted to Professor Kakutani for pointing out an oversight in my original version of this note.

\section{Invariant metrics.}

(1.1) If $G$ is a group with left invariant metric $\rho$ and neutral element e, then $\rho\left(g^{-1}, e\right)=\rho(g, e)$ whenever $g \in G$.

(1.2) Suppose $G$ is a group with left invariant metric $\rho$. Then the following statements are equivalent: (a) $\rho$ is right invariant; (b) $\rho$ is invariant under inversion; (c) $\rho$ is invariant under every inner automorphism of $G$.

Proof. (a) implies (b): $\rho\left(x^{-1}, y^{-1}\right)=\rho\left(x^{-1} x, y^{-1} x\right)=\rho\left(e,\left(x^{-1} y\right)^{-1}\right)$ $=\rho\left(e, x^{-1} y\right)=\rho(x, y)$.

(b) implies (c): $\rho\left(g x g^{-1}, g y g^{-1}\right)=\rho\left(x g^{-1}, y g^{-1}\right)=\rho\left(g x^{-1}, g y^{-1}\right)$ $=\rho\left(x^{-1}, y^{-1}\right)=\rho(x, y)$.

(c) implies (a): $\rho(x g, y g)=\rho\left(g x g g^{-1}, g y g g^{-1}\right)=\rho(g x, g y)=\rho(x, y)$.

Presented to the Society, December 28, 1951; received by the editors October 9, 1951 and, in revised form, October 18, 1951.

1 National Research Fellow.

2 Numbers in brackets indicate references at the end of the paper.

$3 \$ 1$ is closely related to work of van Dantzig $[3, \S \S 3-4]$ and could in part be replaced by references to his paper. However, its present form is better suited to our discussion. 
(1.3) Suppose $G$ is a semi-group with metric $\rho$. Then invariance of $\rho$ implies

$$
\rho(a b, x y) \leqq \rho(a, x)+\rho(b, y) \quad \text { whenever }\{a, b, x, y\} \subset G .
$$

If $G$ is a group, invariance of $\rho$ is equivalent to (\$).

Proof. If $\rho$ is invariant, then

$$
\rho(a b, x y) \leqq \rho(a b, x b)+\rho(x b, x y)=\rho(a, x)+\rho(b, y) .
$$

If (\$) holds and $G$ is a group, then

$$
\rho(g u, g v) \leqq \rho(g, g)+\rho(u, v)=\rho(u, v)=\rho\left(g^{-1} g u, g^{-1} g v\right) \leqq \rho(g u, g v),
$$

so $\rho$ is left invariant. A similar argument shows that $\rho$ is right invariant and completes the proof.

(1.4) Suppose $G$ is a semi-group with invariant metric $\rho,\left(G^{*}, \rho^{*}\right)$ is the metric completion of $(G, \rho)$. Let multiplication in $G^{*}$ be defined by termwise multiplication of Cauchy sequences. Then $G^{*}$ is a semi-group, $\rho^{*}$ is invariant on $G^{*}$, and the natural embedding of $G$ in $G^{*}$ is an isomorphism as well as an isometry. If $G$ is a group, then so is $G^{*}$.

Proof. As usual, two Cauchy sequences $u_{\alpha}$ and $v_{\alpha}$ of $G$ are said to be equivalent $\left(u_{\alpha} \sim v_{\alpha}\right)$ if $\lim _{i} \rho\left(u_{i}, v_{i}\right)=0$. The elements of $G^{*}$ are equivalence classes of Cauchy sequences, with $\rho^{*}\left(\left[x_{\alpha}\right],\left[y_{\alpha}\right]\right)$ $=\lim _{i} \rho\left(x_{i}, y_{i}\right)$. Now suppose $x_{\alpha}, x_{\alpha}^{\prime}, y_{\alpha}, y_{\alpha}^{\prime}$ are Cauchy sequences with $x_{\alpha} \sim x_{\alpha}^{\prime}$ and $y_{\alpha} \sim y_{\alpha}^{\prime}$. For each $n$ let $z_{n}=x_{n} y_{n}$ and $z_{n}^{\prime}=x_{n}^{\prime} y_{n}^{\prime}$. From (\$) above it follows that $z_{\alpha}$ and $z_{\alpha}^{\prime}$ are Cauchy sequences, with $z_{\alpha} \sim z_{\alpha}^{\prime}$. Thus we can define multiplication in $G^{*}$ by $\left[x_{\alpha}\right]\left[y_{\alpha}\right]=\left[\left(x_{1} y_{1}, x_{2} y_{2}, \cdots\right)\right]$ and $G^{*}$ becomes a semi-group in which $G$ is isometrically and isomorphically embedded. It is easy to see that $\rho^{*}$ is invariant on $G^{*}$. And if $G$ is a group, the fact that $G^{*}$ is a group follows from invariance under inversion of $\rho$. The proof is complete.

(1.5) Suppose $G$ is a group having a Hausdorff topology. Then (for $i=1,2)$ the statements $\left(\mathrm{a}_{i}\right)$ and $\left(\mathrm{b}_{i}\right)$ below are equivalent:

$\left(\mathrm{a}_{1}\right) \mathrm{G}$ admits a left invariant metric;

$\left(\mathrm{b}_{1}\right) G$ is first countable at $e$, the group operations are continuous at $e$, and $y g \mid g \in G$ is continuous for each $y \in G$;

$\left(\mathrm{a}_{2}\right) G$ admits an invariant metric;

$\left(b_{2}\right) G$ is a Hausdorff group which admits at e a countable complete system of neighborhoods, each invariant under every inner automorphism of $G$.

Proof. Suppose first that $\left(a_{1}\right)$ holds. Then two of the assertions of $\left(b_{1}\right)$ are obvious. Continuity at $e$ follows for inversion from (1.1) and for multiplication from the inequality,

$$
\rho(x y, e)=\rho\left(y, x^{-1}\right) \leqq \rho(y, e)+\rho\left(e, x^{-1}\right)=\rho(y, e)+\rho(x, e) .
$$


Now suppose $\left(\mathrm{a}_{2}\right)$ holds. That $G$ admits at $e$ a system of neighborhoods of the specified type follows from (1.2), as does the fact that inversion is continuous. Continuity of multiplication follows from $(\$)$ of $(1.3)$.

That $\left(\mathrm{b}_{i}\right)$ implies $\left(\mathrm{a}_{i}\right)$ (for $\left.i=1,2\right)$ follows without difficulty from a general theorem of Kakutani [6] and also from an examination of the proof of Birkhoff [2]. In each case there is a countable complete system $V_{\alpha}$ of neighborhoods of $e$ such that for all $k, V_{k}=V_{\boldsymbol{k}}^{-1}$ and $V_{k}^{3} \subset V_{k-1}$. Assuming $\left(b_{2}\right)$, these can be taken invariant under every inner automorphism of $G$. Then (following Birkhoff) let $\delta(x, y)$ $=\inf _{x}{ }^{-1} y \in V_{k}(1 / 2)^{k}$ and $\rho(x, y)=\inf _{x=u_{0}, u_{n}=y} \sum_{k=1}^{n} \delta\left(u_{k-1}, u_{k}\right)$. In both cases $\rho$ is a left invariant metric compatible with the topology of $G$, and under $\left(b_{2}\right) \rho$ is actually invariant.

2. Complete invariant metrics. An argument essentially the same as that in (2.1) and (2.2) below is used by Mazur and Sternbach $[8$, p. 50$]$ to prove that a $G_{\delta}$ linear subset of a Banach space must actually be closed.

(2.1) Suppose $S$ is a second category topological group and $X$ is a subgroup of $S$. Then $S-X$ is either empty or of second category in $S$.

Proof. Suppose $y \in S-X$. Then $y X \subset S-X$, and if $S-X$ is of first category, so is $y X$; but then so is $X$, and hence $S$ itself, a contradiction completing the proof.

(2.2) Suppose $S$ and $X$ are as in (2.1), and $X$ is a dense $G_{\delta}$ subset of $S$. Then $X=S$.

Proof. We have $X=\bigcap_{i} X_{i}$, where each $X_{n}$ is a dense open set. But then each set $S-X_{n}$ is closed and nowhere dense, so $S-X$ (being the union of these sets) is of first category. The desired conclusion follows from (2.1).

(2.3) Suppose $G$ is a group with invariant metric $\rho$. Then if the space $(G, \rho)$ is topologically complete, $G$ is actually complete under $\rho$.

Proof. Let $\left(G^{*}, \rho^{*}\right)$ be the metric completion of $(G, \rho)$ and recall the facts stated in $(1.4):\left(G^{*}, \rho^{*}\right)$ is a topological group in which $(G, \rho)$ is isomorphically and isometrically embedded. Sierpinski has proved [9] that a topologically complete metric space is a $G_{\delta}$ set relative to every metric space in which it is topologically embedded. Thus from (2.2) it follows that $G$ (as embedded in $G^{*}$ ) is identical with $G^{*}$, and hence $G$ is complete under $\rho$.

Neither (1.4) nor (2.3) is valid if $\rho$ is assumed merely to be leftinvariant. For let $G$ be the group of all homeomorphisms of $[0,1]$ onto itself, with topology supplied by the metric $d(u, v)$ $=\sup _{x \in[0,1]}|u(x)-v(x)|$. Then $G$ is a Hausdorff group and thus admits a left-invariant metric. However, Dieudonné [4] has ob- 
served that $G$ cannot be isomorphically embedded in a complete topological group. (I am indebted to Dr. Ernest Michael for this reference.) It is further easy to see that $G$ is a $G_{\delta}$ set in the set of all continuous monotone functions on $[0,1]$, and that the latter set is complete in the metric $d$. Thus $G$ is topologically complete.

From (2.3) and (1.5) we obtain:

(2.4) Suppose $G$ is a Hausdorff group whose neutral element admits a countable complete system of neighborhoods, each invariant under every inner automorphism of $G$. Then $G$ admits an invariant metric, and if topologically complete must be complete under every invariant metric.

Dr. Michael has pointed out that (2.4) implies:

(2.5) The space of a metric abelian group is topologically complete if and only if it is complete in the uniformity determined by the neighborhoods of the neutral element.

A corollary of (2.4) is:

(2.6) Every complete linear metric space can be metrized as a (complete) space of type (F).

This answers affirmatively a question of Banach $[1$, p. 232]. The question has also been considered by G. G. Lorentz [7]. His principal results are reduced by (2.6) to previous results of other authors.

Another consequence of (2.4) is:

(2.7) A normed linear space is a Banach space if and only if it is topologically complete.

\section{REFERENCES}

1. Stefan Banach, Théorie des opérations linéaires, Warsaw, 1932.

2. Garrett Birkhoff, A note on topological groups, Compositio Math. vol. 3 (1936) pp. $427-430$.

3. D. van Dantzig, Zur topologischen Algebra, I, Math. Ann. vol. 107 (1932) pp. 587-616.

4. Jean Dieudonné, Sur la complétion des groupes topologiques, C. R. Acad. Sci. Paris vol. 218 (1944) pp. 774-776.

5. Shizuo Kakutani, Über die Metrisation der topologischen Gruppen, Proc. Imp. Acad. Japan vol. 12 (1936) pp. 82-84.

6. - Über ein Metrisationproblem, Proceedings of the Physico-Mathematical Society of Japan (III) vol. 20 (1938) pp. 85-90.

7. G. G. Lorentz, Operation in linear metric spaces, Duke Math. J. vol. 15 (1948) pp. $755-761$.

8. S. Mazur and L. Sternbach, Über die Borelschen Typen von linearen Mengen, Studia Math. vol. 4 (1933) pp. 48-53.

9. W. Sierpinski, Sur les ensembles complets d'un espace (D), Fund. Math. vol. 11 (1928) pp. 203-205.

The Institute for Advanced Study 